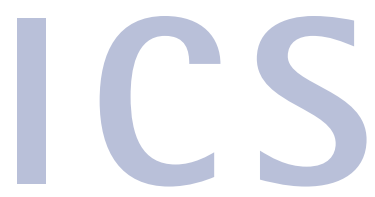

\section{Strong splinting}

Pre-impregnated glass fibre system everStickPerio is specifically designed for all splinting indications including periodontal and post traumatic splints. It is a semimanufactured product consisting of unidirectional glass fibres and a polymer/resin gel matrix for reinforcing dental composites. The fibre reinforced composite is cured directly onto the periodontally compromised teeth, creating a strong but suitably elastic long-term splint that can be easily removed when no longer needed.

It can be used for lingual/palatal splinting, labial splinting, occlusal splinting and post trauma labial splinting. It can also be used as a combined periodontal splint and surface-retained bridge.

Reader response number 54

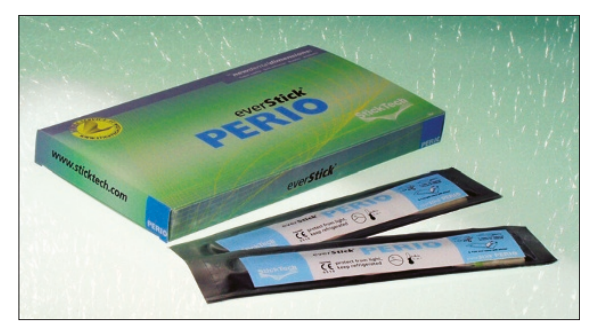

Getting the polished look

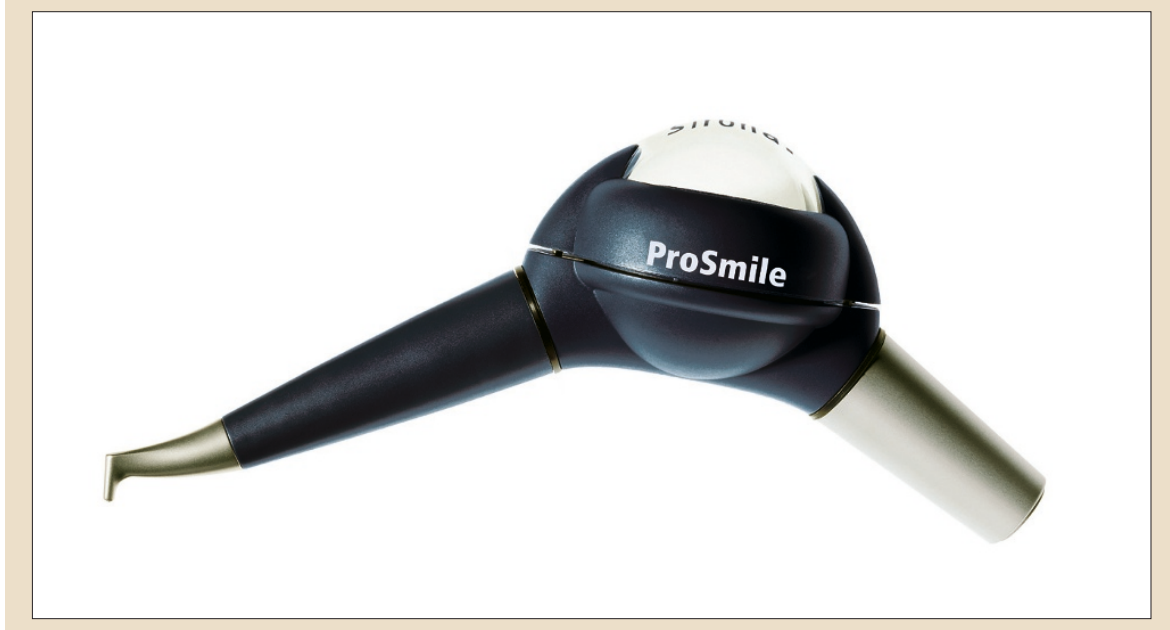

Sirona Dental Systems has added the ProSmile Handy air polishing handpiece to its range. The powder is delivered as a precisely focused jet for the gentle removal of plaque, film and stains from the visible tooth surfaces as well as from fissures and interdental spaces.

The product moulds itself to the user's hand and the handpiece is freely rotat-

\title{
Controlled release delivery system
}

PerioChip is an advanced, controlledrelease, chlorhexidine delivery system that kills and suppresses pocket pathogens for up to three months, according to Dexcel Dental.

The small, thin 'chip' of solid, hydrolysed gelatine, with a cross-linked matrix

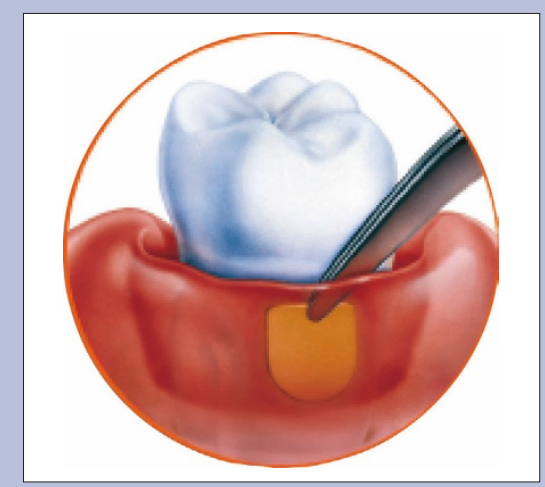

structure when placed into a periodontal pocket gradually biodegrades, releasing bactericidal levels of chlorhexidine over seven to ten days. The product offers an advanced, controlled-release chlorhexidine delivery system and kills and suppresses pocket pathogens for up to three months.

In combination with SRP/debridement, it can reduce pocket depth more than SRP/debridement alone. Used for long-term-maintenance treatment, pocket depth is reduced for at least two years, there is long-term bactericidal action and no induced resistance. Periochip is patient compliance guaranteed and a normal oral hygiene regime is the only requirement after a PerioChip has been inserted.

Reader response number 56 able and can be fitted with either a $120^{\circ}$ or a $90^{\circ}$ nozzle. It is fitted with a Click\&Go interface and is compatible with a wide variety of high-speed handpiece couplings. The large volume chamber means that several patients can be treated in quick succession without the need to replenish the powder.

Reader response number 55

\section{Compact scaler}

The Cavitron Select SPS scaler from Dentsply has a compact design with the boost control placed on the foot pedal. It can, according to the company be of value in deep periodontal pockets where a solution of the clinician's choice can be placed in the reservoir. The handpiece that the scaler inserts into is autoclavable.

Reader response number 57

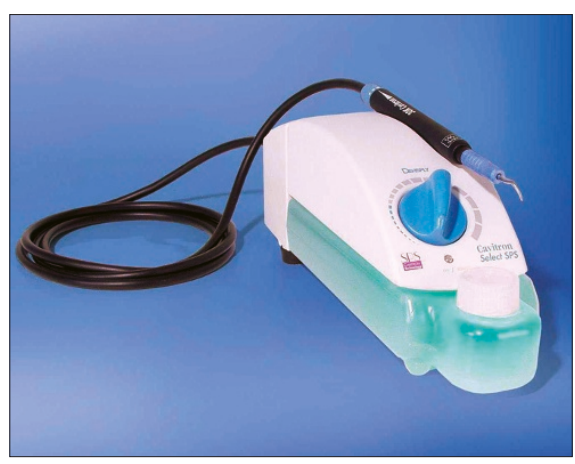




\section{One session therapy}

Pharmaco-mechanical Atridox is a one session therapy for early detected periodontitis and is for use in adults with chronic periodontitis at sites with probing depths of $5 \mathrm{~mm}$ or more, as an adjunct to conventional non-surgical management of periodontal disease. A viscous gel that easily flows into areas difficult to instrument, such as furcations, Atridox lines the pocket and once in contact with the crevicular fluid, precipitates

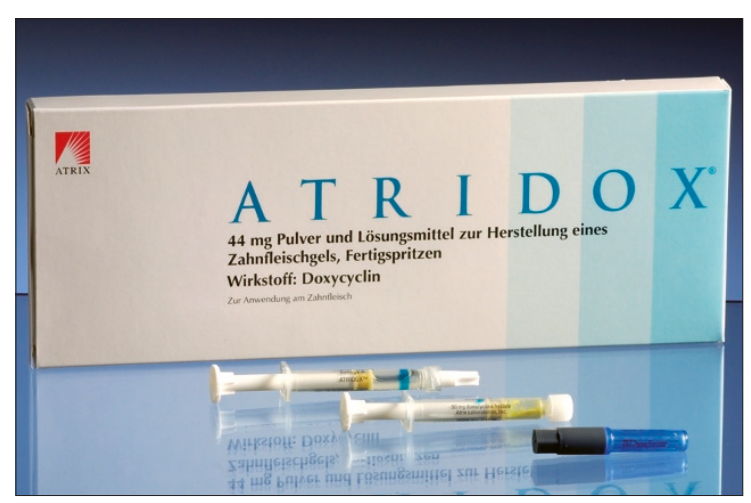
and quickly becomes wax like and remains in the pocket. When the product is applied it releases doxycyline over a prolonged period. The concentration in the pockets reaches several hundred times the MIC90 for the perio-pathogens and it remains at these high levels for at least seven days. Its substantivity helps reduce the micro-organisms residing in these tissues. According to the manufacturers, the clinical result is equivalent to or even better than that achieved with scaling and root planing and hard tissue damage is reduced.

Reader response number 58

\section{Flossing to improve gum health}

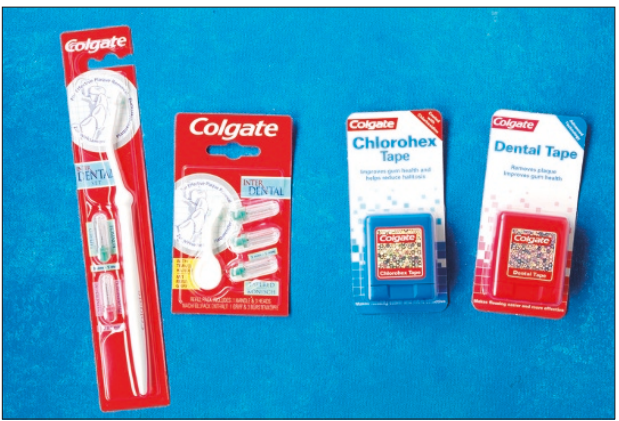

Colgate Flossing Tapes are clinically proven to improve gum health. Manufactured using advanced fibre technology, they deliver more effective interproximal cleaning. The monofilament construction makes them shred, fray and tear resistant and the gliding properties mean they can be used even between tight contact points.

The product is available in two variants including one which contains a chlorhexidine coating.

Reader response number 59

\section{Alternative treatments for periodontitis}

In answer to the growing demand for complimentary products for use within dentistry, the Dental Directory has introduced a range of complementary, alternative and nutritional products designed to strengthen the body's own immune and healing system.

Within the range are several products suitable for treating periodontitis. ThioCare Toothpaste, for example, contains the patented

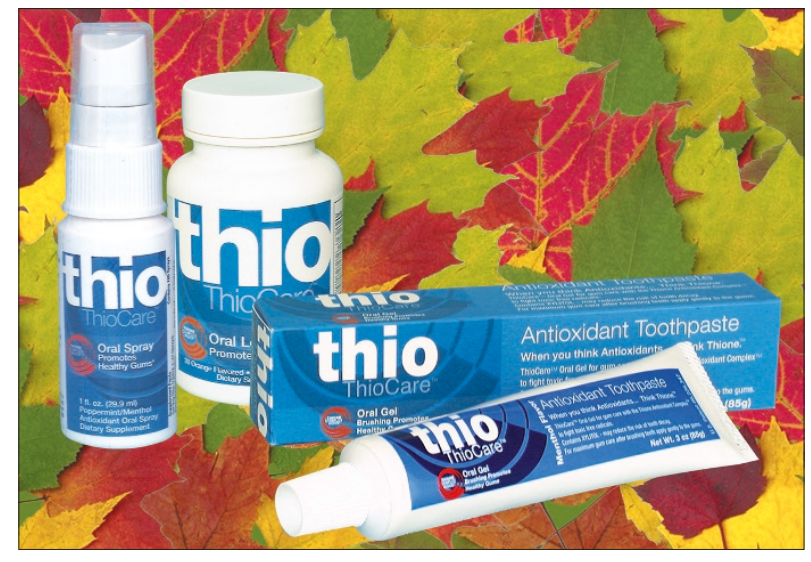
Thione Complex which is a l-gluthathione based formula of antioxidants. The company claims that when delivered topically to the gums, it can reduce free radicals, encourage gum tissue to heal and can be beneficial to those with various forms of gingivitis.

The ThioCare Oral Spray and an Oral Lozenge can also help to maintain healthy gums, while for more serious cases there is the Herbal Mouth and Gum Therapy, which naturally kills bacteria and prevents decay. It contains echinacea, goldenseal and calendula and is formulated without alcohol, artificial sweeteners, chemical preservatives or dyes.

Reader response number 60 